\title{
Genetic variations of EBV-LMP1 from nasopharyngeal carcinoma biopsies: potential loss of $\mathrm{T}$ cell epitopes
}

\author{
Y.L. Tang*, J.H. Lu*, L. Cao, M.H. Wu, S.P. Peng, H.D. Zhou, C. Huang, Y.X. Yang, \\ Y.H. Zhou, Q. Chen, X.L. Li, M. Zhou and G.Y. Li \\ Cancer Research Institute, Central South University, Changsha, Hunan, the People's Republic of China \\ Correspondence to: G.Y. Li, Cancer Research Institute, Central South University, 110\# Xiang-Ya Road, \\ Changsha, Hunan 410078, the People's Republic of China \\ Fax: +86-731-480-5383. E-mail: ligy@xysm.net
}

\begin{abstract}
To find Epstein-Barr virus (EBV) strains with genetic variations of EBV latent membrane protein 1 (EBV-LMP1) from nasopharyngeal carcinoma (NPC), the full-length DNA of LMP1 genes from 21 NPC biopsies obtained in Hunan province in southern China was amplified and sequenced. Our sequences were compared to those previously reported by the Clustal $\mathrm{V}$ method. Results showed that all 21 sequences displayed two amino acid changes most frequently in LMP1 of CD4 ${ }^{+}$T cell epitopes at codons $144(F \rightarrow I, 21 / 21)$ and $212(G \rightarrow S, 19 / 21)$ or $(G \rightarrow N, 2 / 21)$. We also show that type A EBV strain is prevalent in the cases of NPC from Hunan province with a 30-bp 18/21 deletion, and we highlight that this deletion resulted in loss of one of the CD4 ${ }^{+} \mathrm{T}$ cell-restricted epitopes. The other 3 sequences without this deletion all had a change at codon $344(\mathrm{G} \rightarrow \mathrm{D})$. Furthermore, in the major epitope sequence of $C D 8^{+} \mathrm{T}$ cells restricted by HLA-A2, all 21 sequences showed changes at codons $126(\mathrm{~L} \rightarrow \mathrm{F})$ and $129(\mathrm{M} \rightarrow \mathrm{I})$. Our study discovered that one of the 21 sequence variations harbored a new change at codon 131 $(\mathrm{W} \rightarrow \mathrm{C})$, and $5 / 21$ specimens showed another novel change at codon $115(\mathrm{G} \rightarrow \mathrm{A})$ in the major epitope sequence of CD8 ${ }^{+} \mathrm{T}$ cells restricted by HLA-A2. Our study suggests that these sequence variations of NPC-derived LMP1 may lead to a potential escape from host cell immune recognition, protecting latent EBV infection and causing an increase in tumorigenicity.
\end{abstract}

Key words: Genetic variation; Epitope; Immune recognition; Latent membrane protein 1; Tumorigenicity

*These authors contributed equally to this study.

Research supported by the Key National Research Plan of China (2006CB910502, 2006CB910504), the National Science Foundation of China (No. 30330560), China Postdoctoral Science Foundation (No. 20060390264), and Hunan Province Natural Sciences Foundations of China (No. 05JJ300064).

Received June 27, 2007. Accepted December 18, 2007

\section{Introduction}

It is now known that Epstein-Barr virus (EBV) infects more than $90 \%$ of the world's adult population. Upon infection, the individual remains a lifelong carrier of the virus. EBV is strongly associated with the development of several cancers, particularly with Burkitt's lymphoma, Hodgkin's disease, lymphoproliferative disorders, and nasopharyngeal carcinoma (NPC) (1). The clonal EBV genome is consistently detected in invasive carcinomas and highgrade dysplastic lesions $(2,3)$. Such observations imply that latent viral infection may have taken place before the expansion of the malignant cell clone. A current hypothesis proposes that EBV plays a critical role in transforming nasopharyngeal epithelial cells into invasive cancer (4). Latent membrane protein 1 (LMP1) is of special interest since it is generally considered to be the main EBV oncogene $(5,6)$. $\mathrm{EBV}$, in conjunction with environmental and genetic factors, plays a role in the development of NPC.

Recent studies have suggested that some of the sequence variations in the LMP1 gene such as a 30-bp deletion and multiple base substitutions and amino acid changes 
in HLA-A2-restricted cytotoxic T lymphocyte (CTL) epitopes are associated with an increase in tumorigenicity and with a decrease in immune recognition $(7,8)$. HLA-A2-restricted $\mathrm{CD}^{+}$epitopes in LMP1 that are recognized by CTLs from healthy, EBV-seropositive individuals have been identified (9). Data indicate that EBV strains with specific variants of LMP1 are more likely to be found in NPC (10). Furthermore, although previous studies on LMP1 have indicated the existence of NPC-specific strains and variation of the LMP1 gene in some regions in southern China $(6,11)$, this information is not known for Hunan, a province in southern China. Thus, it is critically important to study variations of the LMP1 gene since Hunan is also one of the high-risk areas for NPC (12).

Thus, in the present study, DNA and amino acid sequence analyses were conducted by the Clustal $\mathrm{V}$ method to detect EBV strains with genetic variations of EBV-LMP1 integrated in the NPC genome, to evaluate specific sequence variations within HLA-A2-restricted epitopes and to find EBV strains with specific variants of LMP1 in NPC biopsy specimens obtained from patients from Hunan province. Twenty-one full-length LMP1 genes were amplified and sequenced.

\section{Material and Methods}

\section{Biopsy specimens}

A total of 21 NPC biopsy specimens were collected from patients in Hunan province in southern China, ranging in age from 31 to 64 years. Four were females and 17 were males. A part of each specimen was submitted to histopathological diagnosis of NPC in the Pathology Department of the First Affiliated Hospital of Xiangya Medical School, Central South University, and the remainder was snap-frozen and kept in liquid nitrogen for subsequent DNA analysis.

\section{Isolation of DNA}

Genomic DNA was prepared from 21 NPC biopsy samples as described previously (6). NPC tissues were digested for $16 \mathrm{~h}$ at $55^{\circ} \mathrm{C}$ in a buffer that contained $10 \mathrm{mM}$ Tris- $\mathrm{HCl}, \mathrm{pH}$ 8.0, $100 \mathrm{mM} \mathrm{NaCl}, 25 \mathrm{mM}$ EDTA, and proteinase $\mathrm{K}(0.1 \mathrm{mg} / \mathrm{mL})$. Samples were extracted twice with an equal volume of phenol/chloroform and dialyzed against $10 \mathrm{mM}$ Tris- $\mathrm{HCl}, \mathrm{pH}$ 8.0, and $1 \mathrm{mM}$ EDTA. RNase A (100 $\mu \mathrm{g} / \mathrm{mL}$ ) was added to the dialyzed sample and incubated at $37^{\circ} \mathrm{C}$ for $2 \mathrm{~h}$, followed by phenol/chloroform extraction and dialysis.

Polymerase chain reaction amplification and DNA sequencing

The full-length DNA of the LMP1 gene was amplified by polymerase chain reaction (PCR) using primers P-L-F: 5'TCAGGCAAGCCTATGACATG-3' nucleotide coordination relative to B95-8, 168116-168135 and P-L-R: 5'-TCAGTT GCCTTGCTCCTGCC-3' nucleotide coordination relative to B95-8, 169508-169489. Each PCR experiment included a positive control of DNA from the B95-8 strain and a negative control of $\mathrm{ddH}_{2} \mathrm{O}$ instead of DNA. Reactions were carried out in a thermal cycle (Gene Amp ${ }^{R}$ PCR System 9700, Applied Biosystems, Foster city, CA, USA). Fifty microliters of reaction mixtures of 1.4-kb fragments was purified with the Gel Extraction Mini Kit (Watson Biotechnologies, Inc., Shanghai, China). Purified PCR products were sent to the Invitrogen Company (Shanghai, China) for sequencing.

\section{Phylogenetic analysis}

Nucleotide sequences of LMP1 were translated into amino acid ones corresponding to that of the prototype B95-8 and were aligned by the Clustal $\mathrm{V}$ method using the Megalign software (DNA Star, Inc., Madison, WI, USA). Genetic distance and the phylogenetic tree were determined.

\section{Nucleotide sequence accession numbers}

The nucleotide sequence data of LMP1 from 21 NPC reported in the present paper are available in the GenBank database under accession numbers EF419184-EF419204.

\section{Epstein-Barr virus genotyping}

The primers selected to amplify strain-specific variations in the EBNA-3C gene loci have been previously reported $(11,13)$. Conditions and parameters for PCR amplification of the EBV DNA sequence corresponding to the EBNA-3C gene were as described by Cheung et al. (11). The sizes of the PCR products were analyzed by agarose gel electrophoresis. DNA from B95-8 and Jijoye cells was used for control of the type A strain and type B strain, respectively.

\section{Results}

\section{General sequence characteristics of the LMP1 gene}

The full-length LMP1 gene from biopsies was amplified by PCR and determined by and compared with the sequences of the prototype B95-8, NPC-CAO strain (GenBank accession No. AF304432), and NPC strains (China1, China2, AY601307 (NPC1-Lin)), respectively. Sequence analysis revealed that the LMP1 genes from NPC samples were $82.6-91.7$ and $89.8-99.7 \%$ similar at the amino acid level to those of the B95-8 strain, AY601307 (6), respectively. Pattern 1 reported by Lin et al. (6) is prevalent 
among the cases of NPC found in Hunan Province of Southern China. The full LMP1 sequences from NPC8 and NPC15 were fully identified (100\%) and were found to be the same as the sequence of AY601307, except that the latter has one more copy of an 11-amino acid repeat. The phylogram tree is shown in Figure 1. Loss of an Xhol cut site occurred at the $\mathrm{N}$-terminus (21/21). A high frequency of deletions or mutations or multiple base substitutions leading to amino acid changes in LMP1 from NPC tissues was found in Hunan Province (Figure 2).

\section{Epstein-Barr virus genotyping}

The primers chosen to amplify the EBNA-3C genes encompass strain-specific deletions (13). A representative result was shown in Figure 3. It showed 153-bp fragments from B95-8 and 21 NPC cases, as shown by NPC1-11. The expected size of the PCR product of the type A strain was $153 \mathrm{bp}$, as demonstrated by the B95-8 control, and the expected size of the type B strain was $246 \mathrm{bp}$, as shown by Jijoye cells. All EBV genotypes isolated from 21 NPC cases from Hunan province were type A of the EBV strain.

\section{Sequence variations in CD4+ T cell epitopes}

Leen et al. (14) showed that three peptides in LMP1 were CD4+ T cell epitopes. In the present study, 85.7\% $(18 / 21)$ of the NPC-LMP1 sequences displayed the $30-b p$ deletion, which resulted in loss of the third CD4+ T cell epitope. The three sequences without this deletion all had a change at $344(G \rightarrow D)$. Furthermore, the most frequent amino acid changes in the DNA sequences occurred at two positions: at amino acids $144(\mathrm{~F} \rightarrow \mathrm{I}, 21 / 21)$ and 212 $(\mathrm{G} \rightarrow \mathrm{S}, 19 / 21)$ or $(\mathrm{G} \rightarrow \mathrm{N}, 2 / 21)$, and some sporadic mutations also occurred at other amino acids in the LMP1 of CD4+ T cell epitopes (Table 1).

\section{Analysis of HLA-A2-restricted epitopes}

The DNA sequences of 12 potential HLA-A2-binding peptides (amino acids 32-181) within LMP1 were analyzed (6). DNA sequence changes that resulted in amino acid changes were detected in peptides 1, 2, 5, 8, 10, and 11 (Table 2). Of particular interest was peptide 1, which was identified previously as the major HLA-A2-restricted CTL epitope in LMP1 (9). The DNA sequence of peptide 1 displayed amino acid changes at the following three positions: 126, 129 and 131. At amino acid 126, Leu was substituted by Phe in $100 \%$ (21/21) of EBV isolates from NPC specimens. At amino acid 129, a consistent change from ATG to ATT resulting in an amino acid change from Met to lle was detected in $100 \%(21 / 21)$ of EBV isolates from NPC samples, and NPC4 had a point mutation at residue 131 where TGG (Trp) was simultaneously changed to TGC (Cys). This patient was a 56-year-old man with poorly differentiated nasopharyngeal squamous cell carcinoma. Peptide 10 had an amino acid substituted at residue 115 where GGG (Gly) was changed to GCC (Ala) in $23.8 \%$ $(5 / 21)$ of NPC samples. It was the first time that these variations were reported in the EBV-LMP1 gene from NPC compared with that from the prototype B95-8.

\section{Copy numbers of the 33-bp repeat in latent membrane protein 1}

Previous reports have shown the presence of a varying copy of an 11-amino acid repeat between amino acids 250 and 308 in the carboxy-terminal domain of LMP1. Examination of the 21 DNA sequences revealed that 14 of them had four copies of this repeat, 5 of them had five copies, and 2 had a maximum of seven copies. There was no correlation between copy numbers of repeat elements and disease status $(P>0.05)$.

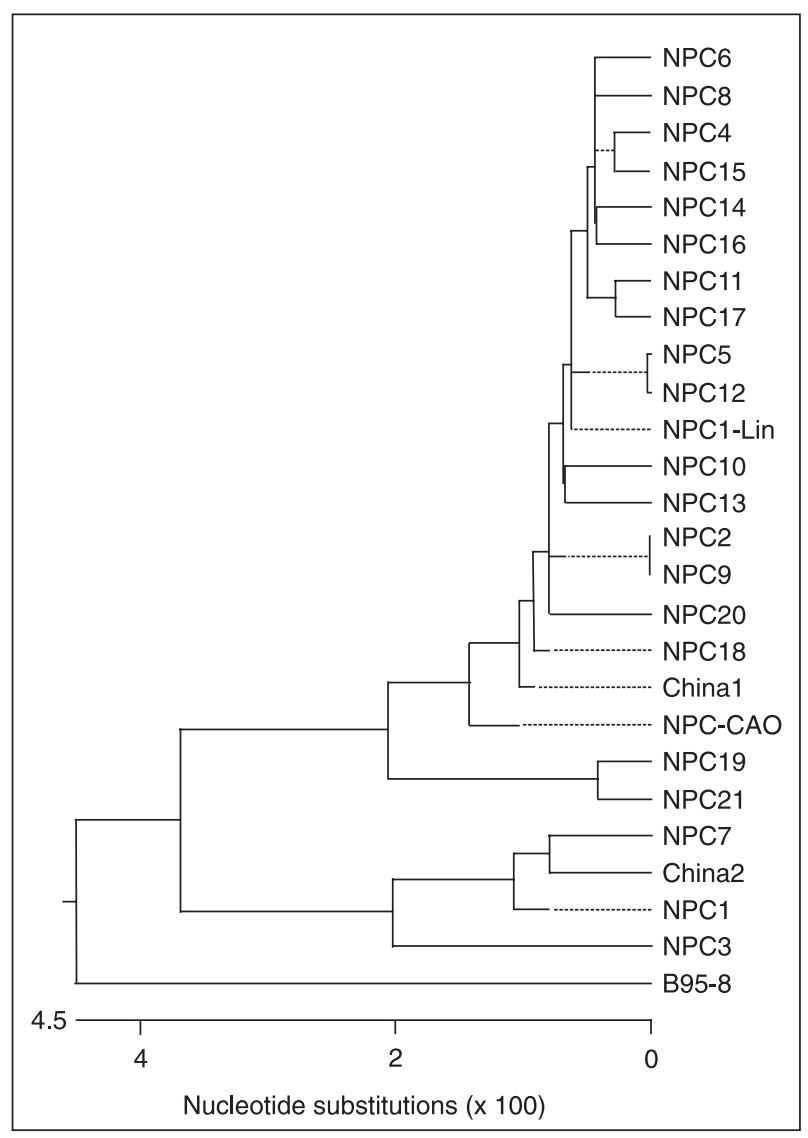

Figure 1. The phylogram tree of the LMP1 gene at the amino acid level from 21 cases of nasopharyngeal carcinoma (NPC; compared with the sequence from the prototype B95-8, NPCCAO, China1, China2, NPC1-Lin). 


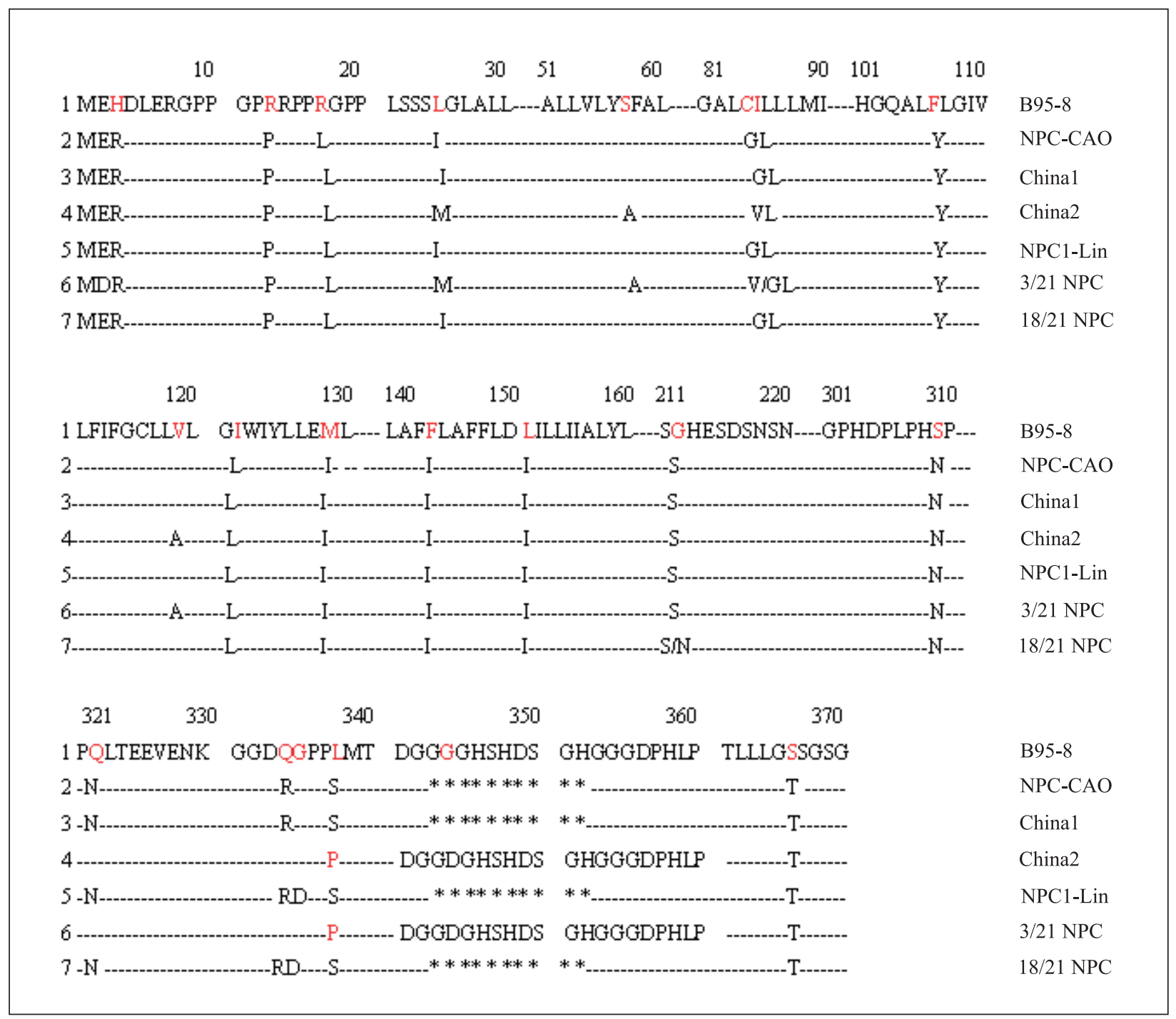

Figure 2. Main variations of full-length amino acid sequences of LMP1 from NPC1-21, B95-8, and 4 previously described. The deletions are indicated by asterisks at codons 343-352. NPC = nasopharyngeal carcinoma.

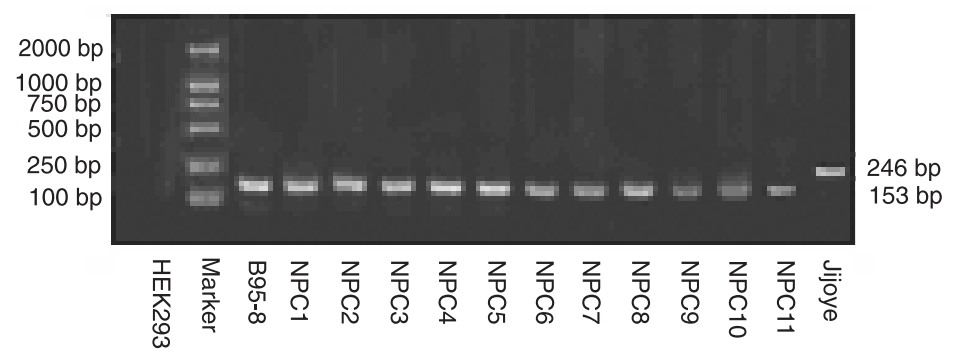

Figure 3. Epstein-Barr virus typing by the EBNA-3C region in primary nasopharyngeal carcinoma (NPC) specimens. 
Table 1. Sequence variation in CD4+ $T$ cell epitopes of latent membrane protein 1 (LMP1) from patients with nasopharyngeal carcinoma.

\begin{tabular}{|c|c|c|c|c|c|}
\hline Peptide & Amino acid residues & Peptide residues & Amino acid position & Codon change & Frequency \\
\hline \multirow[t]{2}{*}{1} & $130-144$ & LWRLGATIWQLLAFF & 131 & $\mathrm{TGG} \rightarrow \mathrm{TGC}(\mathrm{W} \rightarrow \mathrm{C})$ & $1 / 21$ \\
\hline & & & 144 & $\mathrm{TTC} \rightarrow \mathrm{ATC}(\mathrm{F} \rightarrow \mathrm{I})$ & $21 / 21$ \\
\hline \multirow[t]{8}{*}{2} & $211-225$ & SGHESDSNSNEGRHH & 212 & $\mathrm{GGC} \rightarrow \mathrm{AGC}(\mathrm{G} \rightarrow \mathrm{S})$ & $19 / 21$ \\
\hline & & & & $\mathrm{GGC} \rightarrow \mathrm{AAC}(\mathrm{G} \rightarrow \mathrm{N})$ & $2 / 21$ \\
\hline & & & 213 & $\mathrm{CAT} \rightarrow \mathrm{CAG}(\mathrm{H} \rightarrow \mathrm{Q})$ & $1 / 21$ \\
\hline & & & 215 & $\mathrm{TCT} \rightarrow \mathrm{ACT}(\mathrm{S} \rightarrow \mathrm{T})$ & $1 / 21$ \\
\hline & & & 218 & $\mathrm{AAC} \rightarrow \mathrm{GAC}(\mathrm{N} \rightarrow \mathrm{D})$ & $2 / 21$ \\
\hline & & & 220 & $\mathrm{AAC} \rightarrow \mathrm{AGC}(\mathrm{N} \rightarrow \mathrm{S})$ & $2 / 21$ \\
\hline & & & 221 & $\mathrm{GAG} \rightarrow \mathrm{GAC}(\mathrm{E} \rightarrow \mathrm{D})$ & $1 / 21$ \\
\hline & & & 222 & $\mathrm{GGC} \rightarrow \mathrm{GCC}(\mathrm{G} \rightarrow \mathrm{A})$ & $2 / 21$ \\
\hline \multirow[t]{5}{*}{3} & $340-354$ & TDGGGGHSHDSGHGG & $343-352$ & a 30-bp deletion & $18 / 21$ \\
\hline & & & & without a 30-bp deletion & $3 / 21$ \\
\hline & & & 344 & $\mathrm{GGC} \rightarrow \mathrm{GAC}(\mathrm{G} \rightarrow \mathrm{D})$ & $3 / 3$ \\
\hline & & & 354 & $\mathrm{GGT} \rightarrow \mathrm{GCT}(\mathrm{G} \rightarrow \mathrm{T})$ & $2 / 21$ \\
\hline & & & & $\mathrm{GGT} \rightarrow \mathrm{ACT}(\mathrm{G} \rightarrow \mathrm{T})$ & $1 / 21$ \\
\hline
\end{tabular}

Table 2. Sequence variation in the HLA-A2-restricted peptides of latent membrane protein 1 (LMP1) from patients with nasopharyngeal carcinoma.

\begin{tabular}{|c|c|c|c|c|c|}
\hline Peptide & Amino acid residues & Peptide residues & Amino acid position & Codon change & Frequency \\
\hline \multirow[t]{3}{*}{1} & $125-133$ & YLLEMLWRL & 126 & $\mathrm{TTA} \rightarrow \mathrm{TTC}(\mathrm{L} \rightarrow \mathrm{F})$ & $21 / 21$ \\
\hline & & & 129 & ATG $\rightarrow$ ATT $(\mathrm{M} \rightarrow \mathrm{I})$ & $21 / 21$ \\
\hline & & & $131^{*}$ & $\mathrm{TGG} \rightarrow \mathrm{TGC}(\mathrm{W} \rightarrow \mathrm{C})$ & $1 / 21$ \\
\hline 2 & $32-40$ & LLLALLFWL & 33 & $\mathrm{CTC} \rightarrow \mathrm{ATC}(\mathrm{L} \rightarrow \mathrm{I})$ & $3 / 21$ \\
\hline 5 & $173-181$ & WLLLFLAIL & 178 & $\mathrm{CTG} \rightarrow \mathrm{ATG}(\mathrm{L} \rightarrow \mathrm{M})$ & $16 / 21$ \\
\hline 8 & $171-179$ & LLWLLLFLA & 178 & $\mathrm{CTG} \rightarrow \mathrm{ATG}(\mathrm{L} \rightarrow \mathrm{M})$ & $16 / 21$ \\
\hline \multirow[t]{2}{*}{10} & $110-118$ & VLFIFGCLL & 115 & $\mathrm{GGG} \rightarrow \mathrm{GGC}(\mathrm{G} \rightarrow \mathrm{G})$ & $16 / 21$ \\
\hline & & & $115^{*}$ & $\mathrm{GGG} \rightarrow \mathrm{GCC}(\mathrm{G} \rightarrow \mathrm{A})$ & $5 / 21$ \\
\hline 11 & $54-62$ & VLYSFALML & 57 & $\mathrm{TCC} \rightarrow \mathrm{GCC}(\mathrm{S} \rightarrow \mathrm{A})$ & $3 / 21$ \\
\hline
\end{tabular}

${ }^{*}$ These variations are reported here for the first time.

\section{Discussion}

In contrast to the expression of nearly 100 viral genes during replication, EBV-targeted cells express at most 12 latent genes. These genes are usually activated and proliferate to express 6 nuclear antigens, including EBNA-1, -2, $-3(3 A),-4(3 B),-5(L P)$, and -6 (3C), and 3 latent membrane proteins, LMP1, LMP2A, and LMP2B. Along with three kinds of untranslated RNAs, EBV encodes mRNA 1 and mRNA 2, and a family of BamH1A transcripts (15). The limited numbers of viral genes expressed reduce the possibility of recognition of infected cells by CTLs. During the development of NPC, LMP1 is expressed in the absence of the EBNAs, the major viral CTL targets. Malignant cells that express LMP1 are not recognized due to the persis- tence of epitope changes in LMP1, and cells whose LMP1 epitopes are recognized would be eliminated. Our results showed that the pattern 1 reported by Lin et al. (6) is prevalent in NPC cases from Hunan, southern China. High frequencies of deletion and mutation in EBV-LMP1 from NPC tissues were found in Hunan, which is also one of the high-risk areas for NPC (12). The data also indicated that LMP1 sequences from NPC tissues carry mutations which are likely to affect critical functions of the LMP1 gene. LMP1 sequences from NPC isolates significantly increased tumorigenicity when compared to LMP1 sequences from the prototype B95-8 strain or EBV isolates from normal $B$ cells (16), but the exact role of these mutations in enhancing tumorigenicity is not clear.

CD4+ $\mathrm{T}$ cells are primarily known as helper immune 
cells, while a role for CD4+ T cells as direct mediators of effector function was detected in a recent report about the mouse $\gamma$-herpesvirus model $(14,17,18)$. Bickham et al. (19) also revealed the polarized Th1 phenotype (IFN- $\gamma$ ) of EBNA1-specific CD4+ T cells, and reported initial evidence that Th1 CD4+ T cells play several critical roles in host defense against viral infection and tumors. In the present study, $85.7 \%(18 / 21)$ of the NPC-LMP1 sequences displayed the 30 -bp deletion, which resulted in loss of one of the CD4+ T cell-restricted epitopes. In previous reports, the 30-bp deletion was regarded as a biomarker in NPC-LMP1 $(7,8,20,21)$. Interestingly, NPC4 harbored a new change at $131(\mathrm{~W} \rightarrow \mathrm{C})$, which was involved in CD4+ and CD8+ T cellrestricted epitopes. Antigen presentation to CD4+ T helper cells was reduced owing to the potentiation of regulatory $T$ cell function by NPC-LMP1 transfectants, which produce increased levels of interleukin-10, rendering CD4+ T cells hyporesponsive (22). After primary EBV infection, $T$ cells may not recognize activation by NPC-LMP1. These observations have important implications for the establishment of EBV-associated malignancy in the context of infection with tumor-associated EBV-LMP1 variants.

Recent reports have also indicated that variant sequences of CD8+CTL responses can result in poor recognitions by specific T cells $(10,23)$. Polyclonal CD8+ CTLs that recognized the peptide epitopes YLLEMLWRL and YLQQNWWTL from LMP1 in association with HLA-A2 were isolated from seropositive donors (9). Most of the sequence variations in LMP1 detected in our study are consistent with those reported by Lin et al. (6) and Edwards et al. (10) for different EBV strains while the variation in peptide 10 was first reported here (residue $115(G \rightarrow A)$ ). This variation was not associated with clinical stage, patient age or gender $(P>0.05)$ in the limited samples. Furthermore, the results also demonstrated that the LMP1 sequence in EBV strains present in NPC patients from Hunan differed from that of $\mathrm{B} 95-8$, and that the main A2- restricted $T$ cell epitope (amino acids 125-133: YLLEMLWRL) showed variations which were also present in patients from other regions (6). This variant sequence is not recognized by epitope-specific T cells; this correlates with reduced binding of the variant peptide sequence to HLA-A2 molecules (24). The prevalence of an HLA-A2restricted 'epitope loss variant' of LMP1 in a southern Chinese population with a high A2 prevalence may therefore contribute to immune escape by malignant cells that express LMP1. Functional assays of the CD8+ epitope variant showed that EBV isolates from NPC were dominated by an HLA-A2-restricted 'epitope-loss variant' of LMP1, which would allow the virus to resist immune recognition and may contribute in part to the prevalence of NPC in these populations (25).

We have obtained a panel of data about full-length LMP1 from the NPC high-risk area of Hunan province, and found some novel variations in this study. Although these novel variations were found in the limited number of samples, they are involved in changes of several potential $\mathrm{T}$ cell epitopes. These variations may help some EBV strains to obtain stronger immune resistance in their evolution. The striking consistent sequence variations of LMP1, especially in CD4+ T cell epitopes and HLA-A2-restricted CD8+ CTL epitopes, may contribute to the transformation of epithelial cells in NPC by reducing immune recognition and inducing tumorigenic activities and also by molecular differences and biological properties still to be elucidated. Of course, at present, strain identification based on the data from a limited region in LMP1 and these preferential infections cannot be solely attributed to this difference. Functional analysis of LMP1 is underway and biological assays such as transfection experiments are further required to clarify the roles of each LMP1 variant in cell proliferative diseases. We have described a new technology of infectious cloning for the study of gene function in the Epstein-Barr virus genome (26).

\section{References}

1. Thompson MP, Kurzrock R. Epstein-Barr virus and cancer. Clin Cancer Res 2004; 10: 803-821.

2. Raab-Traub N, Flynn K. The structure of the termini of the Epstein-Barr virus as a marker of clonal cellular proliferation. Cell 1986; 47: 883-889.

3. Raab-Traub N. Epstein-Barr virus in the pathogenesis of NPC. Semin Cancer Biol 2002; 12: 431-441.

4. Lo KW, To KF, Huang DP. Focus on nasopharyngeal carcinoma. Cancer Cell 2004; 5: 423-428.

5. Hu LF, Zabarovsky ER, Chen F, Cao SL, Ernberg I, Klein G, et al. Isolation and sequencing of the Epstein-Barr virus

BNLF-1 gene (LMP1) from a Chinese nasopharyngeal carcinoma. J Gen Virol 1991; 72 (Part 10): 2399-2409.

6. Lin JC, Cherng JM, Lin HJ, Tsang CW, Liu YX, Lee SP. Amino acid changes in functional domains of latent membrane protein 1 of Epstein-Barr virus in nasopharyngeal carcinoma of southern China and Taiwan: prevalence of an HLA-A2-restricted 'epitope-loss variant'. J Gen Virol 2004; 85: 2023-2034.

7. Nagamine $M$, Takahara $M$, Kishibe $K$, Nagato $T$, Ishii $H$, Bandoh N, et al. Sequence variations of Epstein-Barr virus LMP1 gene in nasal NK/T-cell lymphoma. Virus Genes 
2007; 34: 47-54.

8. Saechan V, Mori A, Mitarnun W, Settheetham-Ishida W, Ishida T. Analysis of LMP1 variants of EBV in Southern Thailand: evidence for strain-associated T-cell tropism and pathogenicity. J Clin Virol 2006; 36: 119-125.

9. Khanna R, Burrows SR, Nicholls J, Poulsen LM. Identification of cytotoxic $T$ cell epitopes within Epstein-Barr virus (EBV) oncogene latent membrane protein 1 (LMP1): evidence for HLA-A2 supertype-restricted immune recognition of EBV-infected cells by LMP1-specific cytotoxic T lymphocytes. Eur J Immunol 1998; 28: 451-458.

10. Edwards RH, Sitki-Green D, Moore DT, Raab-Traub N. Potential selection of LMP1 variants in nasopharyngeal carcinoma. J Virol 2004; 78: 868-881.

11. Cheung ST, Leung SF, Lo KW, Chiu KW, Tam JS, Fok TF, et al. Specific latent membrane protein 1 gene sequences in type 1 and type 2 Epstein-Barr virus from nasopharyngeal carcinoma in Hong Kong. Int J Cancer 1998; 76: 399-406.

12. Xiong $\mathrm{W}$, Zeng ZY, Xia JH, Xia K, Shen SR, Li XL, et al. A susceptibility locus at chromosome $3 p 21$ linked to familial nasopharyngeal carcinoma. Cancer Res 2004; 64: 19721974.

13. Lin JC, Lin SC, De BK, Chan WP, Evatt BL, Chan WC. Precision of genotyping of Epstein-Barr virus by polymerase chain reaction using three gene loci (EBNA-2, EBNA$3 C$, and EBER): predominance of type $A$ virus associated with Hodgkin's disease. Blood 1993; 81: 3372-3381.

14. Leen A, Meij P, Redchenko I, Middeldorp J, Bloemena E, Rickinson A, et al. Differential immunogenicity of EpsteinBarr virus latent-cycle proteins for human CD4(+) T-helper 1 responses. J Virol 2001; 75: 8649-8659.

15. Ohga S, Nomura A, Takada H, Hara T. Immunological aspects of Epstein-Barr virus infection. Crit Rev Oncol Hematol 2002; 44: 203-215.

16. Hu LF, Chen F, Zheng X, Ernberg I, Cao SL, Christensson $B$, et al. Clonability and tumorigenicity of human epithelial cells expressing the EBV encoded membrane protein LMP1. Oncogene 1993; 8: 1575-1583.

17. Liu H, Andreansky S, Diaz G, Hogg T, Doherty PC. Reduced functional capacity of CD8+ $T$ cells expanded by post-exposure vaccination of gamma-herpesvirus-infected
CD4-deficient mice. J Immunol 2002; 168: 3477-3483.

18. Thomas PG, Keating R, Hulse-Post DJ, Doherty PC. Cellmediated protection in influenza infection. Emerg Infect Dis 2006; 12: 48-54.

19. Bickham K, Munz C, Tsang ML, Larsson M, Fonteneau JF, Bhardwaj N, et al. EBNA1-specific CD4+ T cells in healthy carriers of Epstein-Barr virus are primarily Th1 in function. $J$ Clin Invest 2001; 107: 121-130.

20. Cheung ST, Lo KW, Leung SF, Chan WY, Choi PH, Johnson PJ, et al. Prevalence of LMP1 deletion variant of EpsteinBarr virus in nasopharyngeal carcinoma and gastric tumors in Hong Kong. Int J Cancer 1996; 66: 711-712.

21. Leung SY, Yuen ST, Chung LP, Chan AS, Wong MP. Prevalence of mutations and 30-bp deletion in the C-terminal region of Epstein-Barr virus latent membrane protein-1 oncogene in reactive lymphoid tissue and non-nasopharyngeal EBV-associated carcinomas in Hong Kong Chinese. Int J Cancer 1997; 72: 225-230.

22. Pai S, O'Sullivan B, Abdul-Jabbar I, Peng J, Connolly G, Khanna R, et al. Nasopharyngeal carcinoma-associated Epstein-Barr virus-encoded oncogene latent membrane protein 1 potentiates regulatory T-cell function. Immunol Cell Biol 2007; 85: 370-377.

23. Stevenson PG. Immune evasion by gamma-herpesviruses. Curr Opin Immunol 2004; 16: 456-462.

24. Duraiswamy J, Burrows JM, Bharadwaj M, Burrows SR, Cooper L, Pimtanothai N, et al. Ex vivo analysis of T-cell responses to Epstein-Barr virus-encoded oncogene latent membrane protein 1 reveals highly conserved epitope sequences in virus isolates from diverse geographic regions. $J$ Virol 2003; 77: 7401-7410.

25. Lin HJ, Cherng JM, Hung MS, Sayion Y, Lin JC. Functional assays of HLA-A2-restricted epitope variant of latent membrane protein 1 (LMP-1) of Epstein-Barr virus in nasopharyngeal carcinoma of Southern China and Taiwan. J Biomed Sci 2005; 12: 925-936.

26. Tang YL, Lu JH, Wu MH, Huang C, Cao L, Peng SP, et al. Lytic replication and induction to production visualization recombinant Epstein Barr virus. Progr Biochem Biophys 2007; 34: 418-424. 
This article has received corrections in agreement with the ERRATUM published in Volume 41 Number 4. 